\title{
Beta Decay Rates Evaluated Within the Gross Theory Using Experimental Data for the Gamow-Teller Resonance Parameter
}

\author{
M. Rocha de Azevedo ${ }^{1}$ • R. C. Ferreira ${ }^{1}$ • C. A. Barbero ${ }^{2,3}$
}

Received: 16 January 2020 / Published online: 10 June 2020

(C) Sociedade Brasileira de Física 2020

\begin{abstract}
We improve the results for the $\beta^{-}$-decay rates obtained within the context of the Gross Theory of Beta Decay (GTBD) by using new values of the parameter $\sigma_{N}$ related to the standard deviation for the Gamow-Teller resonance: we include experimental data when they are available and, if not, we adopt the value of the nearest neighbor previous (that is, with less mass) that has experimental data. We evaluate the $\beta^{-}$-decay rates using a Gaussian energy distribution function with the axial-vector weak coupling constant $\left|g_{A}\right|=1$, considering updated experimental mass defects and also an improved approximation for the Fermi function. Our sample consists of 94 nuclei with mass in the range $46<A<70$, all of them decaying by means of allowed transitions, which are of interest in the pre-supernova phase and have experimental data in terrestrial conditions available in the Letter of Nuclides. We compare our result with those obtained within the same GTBD but using values of $\sigma_{N}$ adjusted in Possidonio (Braz. J. Phys. 48:485, 2018) by minimizing the $\chi^{2}$-function, and also with systematic microscopic calculations. We have shown that the substitution of the adjusted parameters by experimental data in GTBD improves the results for $\beta^{-}$-decay rates by $6.4 \%$.
\end{abstract}

Keywords Beta decay · Adjustment parameters · Gross theory of beta decay

\section{Introduction}

One of the great challenges of nuclear physics has been to develop theoretical models that can reproduce as faithfully as possible the experimental results of the various naturally occurring reactions. Among them, $\beta$-decay and electron capture are of particular interest since they are relevant in astrophysical processes in the pre-supernova stage. Among the various nuclear models existing in the literature for $\beta$ decay, we choose to use the Gross Theory of Beta Decay (GTBD) in this work because it has advantages when working with a large number of nuclei, thus facilitating

C. A. Barbero

cab@fisica.unlp.edu.ar

1 Departamento de Ciências Exatas e Naturais, Universidade Estadual do Sudoeste da Bahia, BR 415 Km 03, Itapetinga, BA, Brazil

2 Departamento de Física, Universidad Nacional de La Plata, C. C. 67, 1900 La Plata, Argentina

3 Instituto de Física La Plata, CONICET, 1900 La Plata, Argentina the computational work (see Refs. [1-3] and references therein).

The GTBD is a nuclear model that takes into account rough approximations of the final energy states of the decaying nucleon, and it is essentially a parametric model for nuclear disintegration rates, which combines arguments of independent particle associated with the Fermi gas model. Thus, the GTBD is a model which associates two other major nuclear models with statistical arguments in a phenomenological way. This is done when the $\beta$-amplitude function of the independent particle model is combined with the density levels of the Fermi gas model corrected to take into account the pairing and shell effects. The contributions in the final part of the resonance (GamowTeller) are included in a parametric way. The model was developed originally by Takahashi and Yamada in 1969 [4] and has undergone several modifications over the years. The many improvements in the model motivated us to look for other ways to contribute to obtaining even more accurate theoretical results when compared with the experimental ones. In this context, we remark that the adjustment parameter $\sigma_{N}$ related to the standard deviation for the Gamow-Teller resonance and used so far in Ref. [1, $2]$, was obtained by minimization of the $\chi^{2}$-function. The 
values obtained in this way correspond to an average value for each $N-Z$ parity. They were calculated for the data sample composed of 94 isotopes of the families of iron, cobalt, nickel, manganese, chromium, copper, titanium and scandium, including all isotopes of these families that decay spontaneously via $\beta^{-}$-decay through allowed transitions (we imposed the condition $\log f t<6$ ) with mass in the range $46<A<70$, which are of interest in astrophysical studies. The results obtained in this way for $\sigma_{N}$ were shown in Table 1 from [1]: 8.14 for odd-odd nuclei, 7.48 for odd-even, 7.7 for even-even and 6.6 for even-odd ones.

The arguments mentioned above motivate us to analyze in the present work how the substitution of the adjustment parameters $\sigma_{N}$ by their experimental values modifies the $\beta^{-}$-decay rates when they are evaluated in the context of the same version of the GTBD from Ref. [1]. The paper is organized as follows. The formalism is briefly summarized in Section 2, our results are presented and discussed in Section 3, and final remarks are drawn in Section 4.

\section{Formalism}

We have adopted the GTBD version from Ref. [1]. We have evaluated the $\beta^{-}$-decay rates considering the new relevant modifications within the GTBD scheme, namely: for the $Q$-values we used the updated experimental data present in the Letter of Nuclides [5], the Fermi function has been calculated following the improved approximation from Aufderheide et al. [6], we have used the Gaussian energy distribution function and for the axial-vector weak coupling constant we have used $\left|g_{A}\right|=1$ [1]. We have selected only the value $\left|g_{A}\right|=1$ for the axial-vector coupling constant because we have shown in Ref. [1] that other values of $\left|g_{A}\right|$ in the interval $[0.76,1.26]$, with the $\sigma_{N}$ values determined by minimization of the $\chi^{2}$-function (shown in Table 1 from Ref. [1]) lead to worst results for the decay rates when compared with experimental data. Similarly, we have selected the Gaussian form for the energy distribution function, $D_{\Omega}(E)$, because we have shown that this one leads to better results than the Exponential, Lorentzian and Modified Lorentzian forms. Thus, when only allowed Fermi $(F)$ and Gamow-Teller $(G T)$ transitions are considered, the Fermi golden rule allows to evaluate the total $\beta^{-}$-decay rate as (in natural units $\hbar=c=m_{e}=1$ )

$$
\begin{aligned}
\lambda_{B D} & =\frac{G_{F}^{2}}{2 \pi^{3}} \int_{-Q}^{0}\left[g_{V}^{2} D_{F}(E)+3 g_{A}^{2} D_{G T}(E)\right] \\
& \times N_{1}\left[1-\left(1-\frac{Q+E}{\varepsilon_{F}}\right)^{\frac{3}{2}}\right] f(Z+1,-E) d E,
\end{aligned}
$$

where $G_{F}=(3.034545 \pm 0.00006) \times 10^{-12}$ n.u. is the weak Fermi coupling constant, $g_{V}=-g_{A}=1$ are the vector and axial-vector effective coupling constants, respectively, $-E>0$ appears because we integrate over the final energy states, $\varepsilon_{F}$ is the nucleon Fermi energy (see Eq. (8) from [1]) $N_{1}$ is the number of neutrons of the parent nuclei, and $Q=$ $M_{P}-M_{D}-1$ with $M_{P}$ and $M_{D}$ being the masses of parent and daughter nuclei, respectively, and $f(Z+1,-E)$ is the integrated Fermi function defined in Eq. (2) from [1]. For the energy distribution function $D_{\Omega}(E)$, which measures the probability that a nucleon undergoes a $\beta$-transition, we assume here a Gaussian form [1, 4]

$D_{\Omega}(E)=\frac{1}{\sqrt{2 \pi} \sigma_{\Omega}} e^{\frac{-\left(E-E_{\Omega}\right)^{2}}{2 \sigma_{\Omega}^{2}}}, \Omega=F, G T$

where $E_{\Omega}$ is the resonance energy and $\sigma_{\Omega}$ the standard deviation. Following the original work from Ref. [4], we assume the nuclei as an uniform charged sphere with radius $1.2 \times A^{\frac{1}{3}} \mathrm{fm}$, which allows to consider the Coulombian (c) displacement of independent particle such as

$E_{F}=E_{c}= \pm\left(1.44 Z_{1} A^{-\frac{1}{3}}-0.7825\right) M e V$,

$\sigma_{F}=\sigma_{c}=0.157 Z_{1} A^{-\frac{1}{3}}$,

where $Z_{1}$ is the proton number of the parent nuclei. For the $G T$ resonance, we use the approximation [7, 8]

$E_{G T}=E_{F}+26 A^{-\frac{1}{3}}-\frac{18.5(N-Z)}{A} \mathrm{MeV}$,

and

$\sigma_{G T}=\sqrt{\sigma_{F}^{2}+\sigma_{N}^{2}}$

with $\sigma_{N}$ being a parameter which comes from the energy propagation produced by the forces dependent of the nuclear spin. In order to set this parameter, we have used here two different methods:

- Method I: We follow the procedure of Refs. [1, 9] to determine $\sigma_{N}$ through the minimization of the function $\chi^{2}=\sum_{n=1}^{N_{0}}\left[\frac{\log \left(\tau_{1 / 2}^{\text {cal }}(n) / \tau_{1 / 2}^{\exp }(n)\right)}{\Delta \log \left(\tau_{1 / 2}^{\exp }(n)\right)}\right]^{2}$,

where $\tau_{1 / 2}^{c a l}$ represents the theoretical value for the halflife calculated with our GTBD, $\tau_{1 / 2}^{\exp }$ is the experimental value taken from the Letter of Nuclides [5], $N_{0}$ is the number of experimental $\beta^{-}$-decaying nuclei, fulfilling the conditions: (i) the branching ratio of the allowed transitions exceeds $\sim 50 \%$ of the total $\beta^{-}$-decay branching ratio and (ii) the ground-state $Q$-value is $\geq 10 A^{-1 / 3} \mathrm{MeV}$

$$
\begin{aligned}
\Delta \log \left(\tau_{1 / 2}^{\exp }(n)\right) & =\log \left[\tau_{1 / 2}^{\exp }(n)+\delta \tau_{1 / 2}^{\exp }(n)\right] \\
& -\log \left[\tau_{1 / 2}^{\exp }(n)\right],
\end{aligned}
$$


with $\delta \tau_{1 / 2}^{\exp }(n)$ being the experimental error. This $\chi^{2}-$ function reinforces the contributions of data with small experimental errors. Moreover, we perform different fittings for even $N$-even $Z\left(N_{0}=17\right)$, odd $N$ - odd $Z\left(N_{0}=28\right)$, odd $N$-even $Z\left(N_{0}=29\right)$ and even $N$-odd $Z\left(N_{0}=20\right)$ nuclei. Needless to say that for $\tau_{1 / 2}^{\text {exp }}$ we use recent data from [5], instead of those that were available when the GTBD was formulated [4]. The results obtained with the GTBD using the $\sigma_{N}$ values determined by means of this method will be hereinafter referred to as GTBD1.

- Method II: In the second case, we use experimental information on $G T$ resonance to fix the parameter $\sigma_{N}$
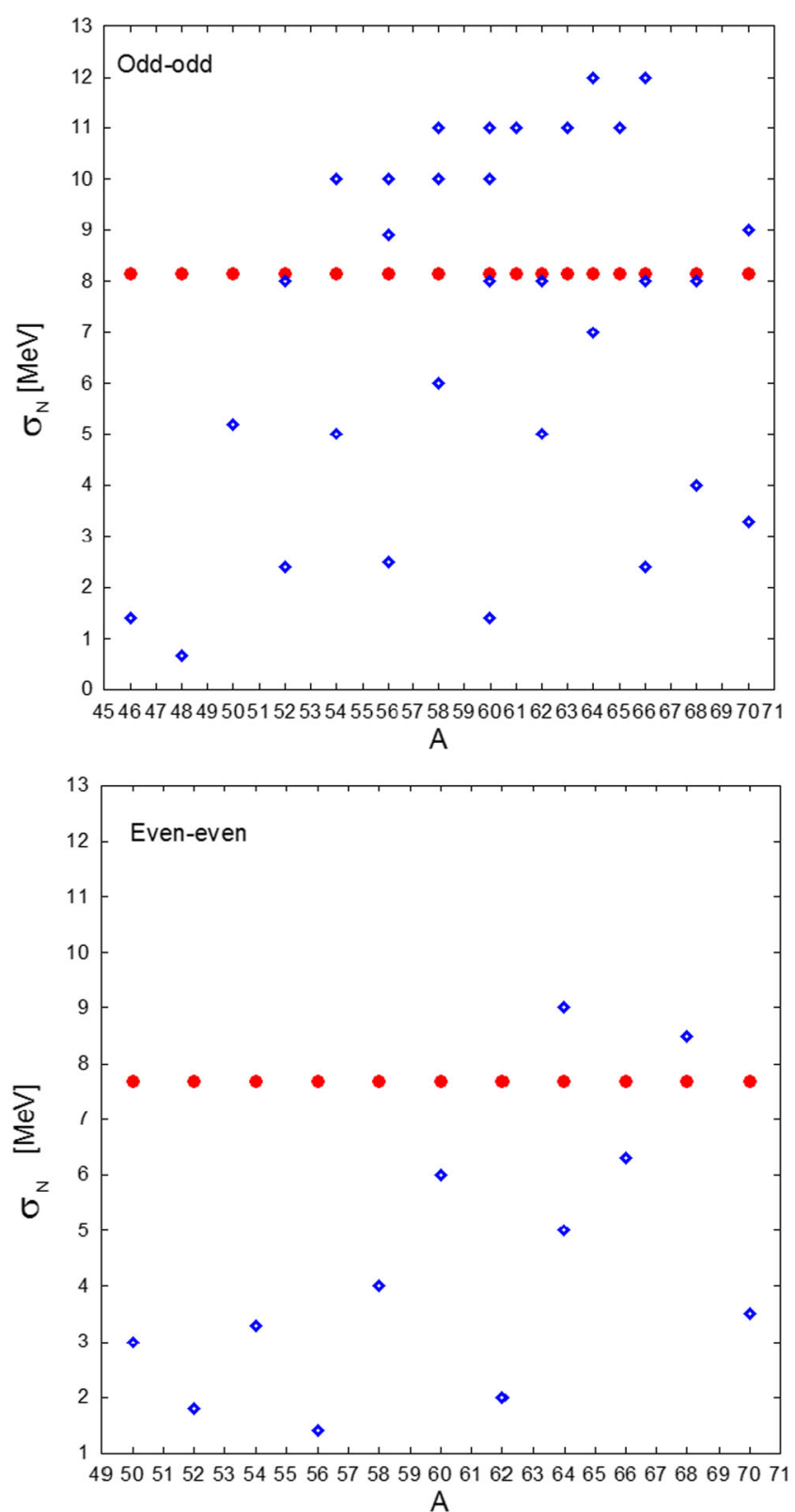

thorough the (6). In this way we have obtained the $\sigma_{N}$ values for those elements of our sample that had experimental data available and only for some exceptions we used data from their nearest neighbors previous (that is, with less mass) that has data. We remark that a subset of 65 nuclei of our sample has experimental data, which corresponds to $\sim 70 \%$ of the complete set, and for the remaining 29 elements we have used data from their closest neighbors. We consider that this is a subset representative of the total sample. Specifically, the nuclei with experimental data were: ${ }^{47,49,50} \mathrm{Ca}$, ${ }^{60-69} \mathrm{Co},{ }^{55-58,60,61,64} \mathrm{Cr},{ }^{59,61-65,68} \mathrm{Fe},{ }^{56-62,64} \mathrm{Mn}$, ${ }^{65,67,69,70} \mathrm{Ni}, \quad{ }^{46-52,54} \mathrm{Sc}, \quad{ }^{51-55,57} \mathrm{Ti}, \quad{ }^{52-58,61} \mathrm{~V}$ and
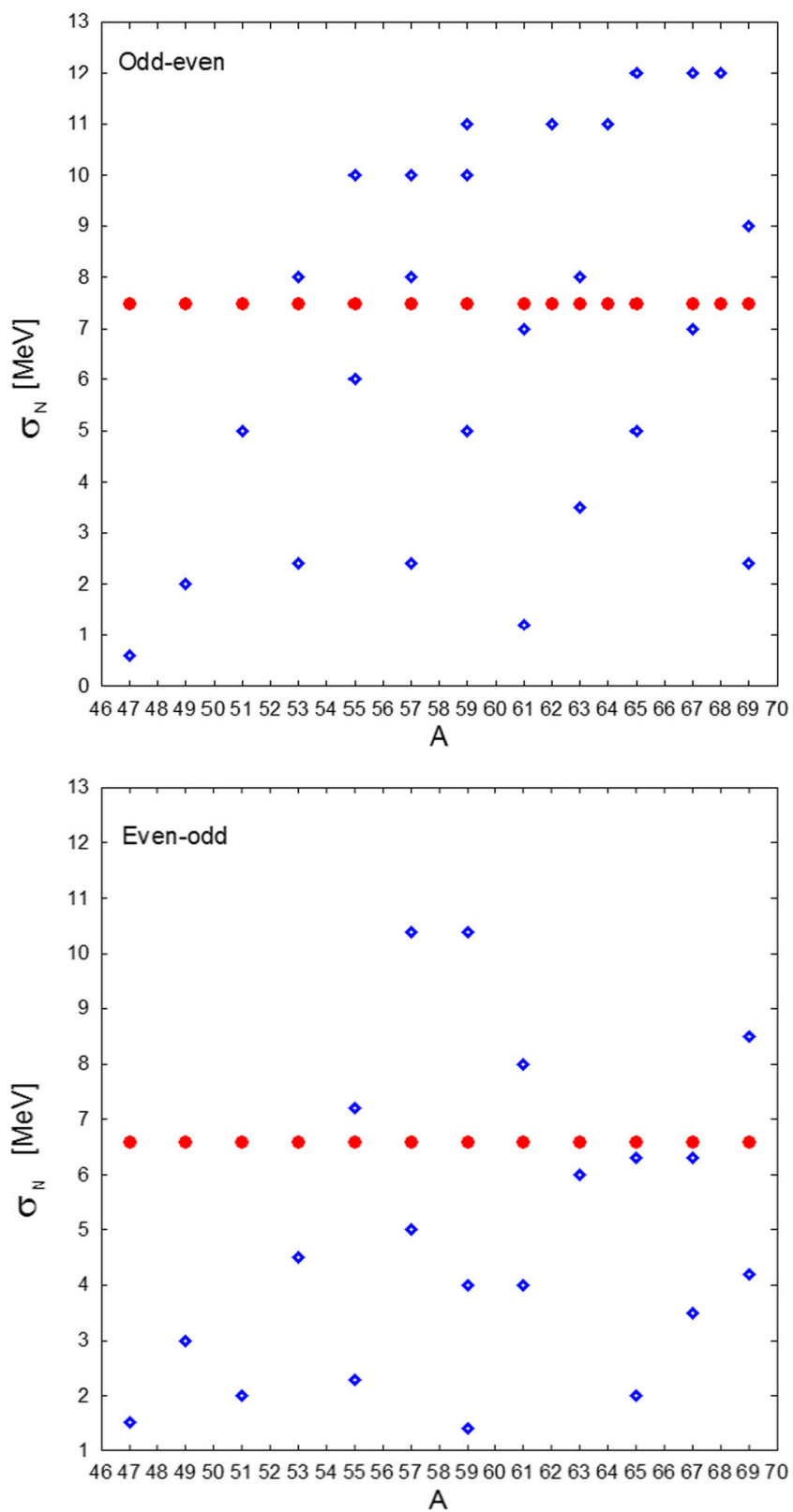

Fig. 1 Values of the $\sigma_{N}$ parameter within Method I (red circles) and Method II (blue squares) 
Fig. 2 Comparison of $\beta^{-}$ half-lives for two different calculations within GTDB model (red circles correspond to GTBD1 and green crosses to GTBD2) for the complete sample of 94 nuclei. Experimental data from Letter of Nuclide [5]

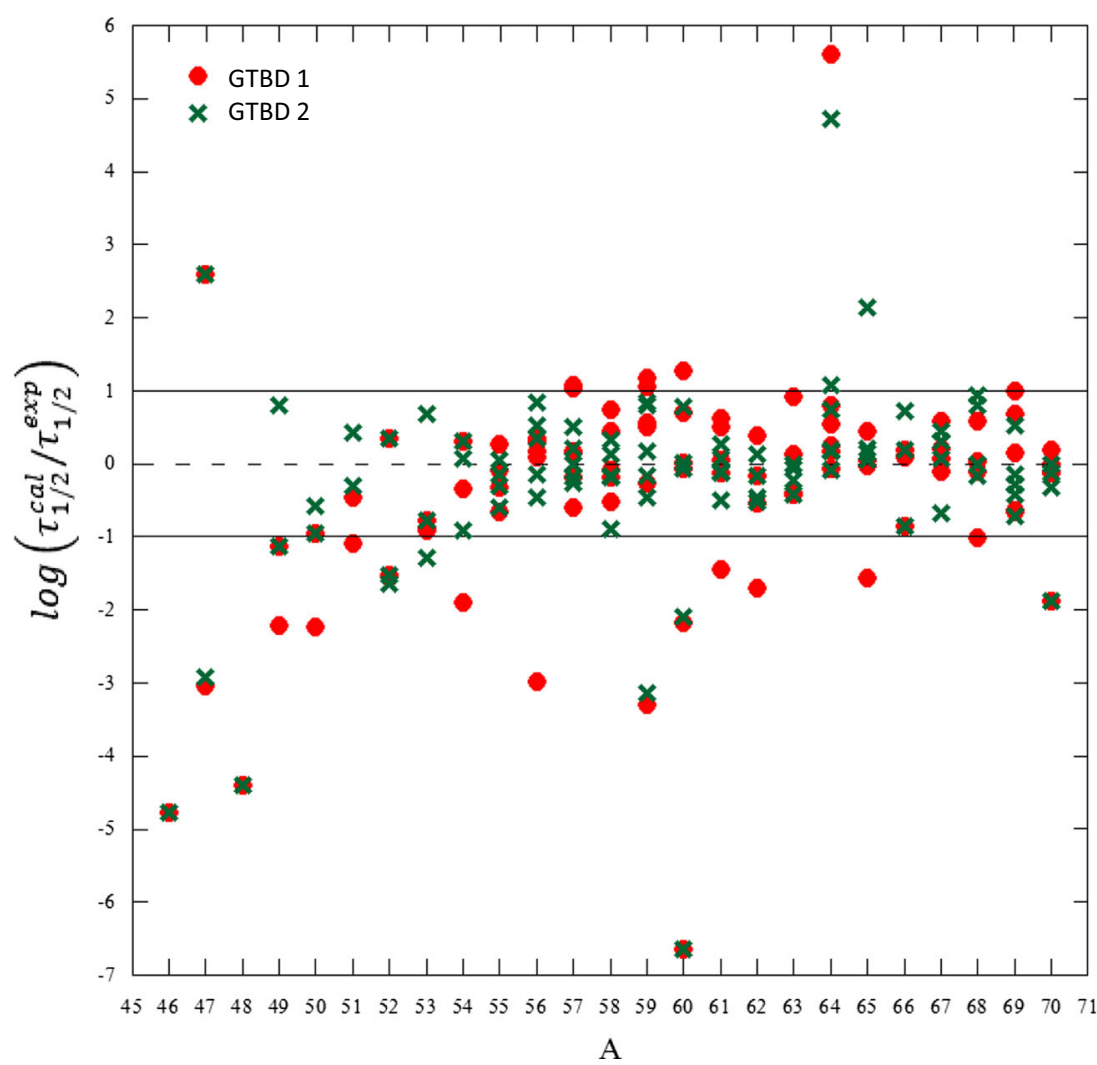

${ }^{66,68-70} \mathrm{Cu}$. Additionally, the nuclei for which we use data from their closest neighbors were: ${ }^{70} \mathrm{Co},{ }^{59} \mathrm{Cr}$, $66,67,69,70 \mathrm{Fe},{ }^{63,65-68} \mathrm{Mn},{ }^{68} \mathrm{Ni},{ }^{53,55-60} \mathrm{Sc},{ }^{56,58-60} \mathrm{Ti}$, $59,60,62-65 \mathrm{~V}$. The results obtained with the GTBD using experimental information to fix the parameter $\sigma_{N}$ will be hereinafter referred to as GTBD2.

\section{Results and Discussions}

Firstly, we show in Fig. 1 the values for the parameter $\sigma_{N}$ obtained within both methods previously described. As mentioned, they have been obtained for a set of 94 nuclei belonging to the cobalt, chrome, copper, iron, manganese, nickel, scandium, titanium, and vanadium families, of interest in astrophysics, in the region mass $46 \leq A \leq 70$. The values obtained with Method I, already given in Table 1 from [1] and repeated above, correspond to an average value (remember that they were obtained by minimizing the $\chi^{2}$-function for each group of nuclei with the same parity separately, which gives a different constant value for each group). On the contrary, the values of $\sigma_{N}$ within Method II vary for the different nuclear species within each parity group, according to the experimental information available for the Gamow-Teller resonance, and for that reason we hope it will lead us to an improvement in the results for the theoretically calculated half-lives.

In Fig. 2 we compare the experimental data for the halflives, $\tau_{1 / 2}^{\text {exp }}$, with the calculated ones, $\tau_{1 / 2}^{c a l}$, both for the GTBD1 and GTBD2 calculations, for the complete set of 94 nuclei of our sample. The results between the lines corresponding to the values -1 and 1 in the graph indicate those nuclei in which the theoretical value differs from the experimental one in less than one order of magnitude. Therefore, GTDB1 gives 73 isotopes within this range and 21 outside while GTDB2 shows 79 isotopes within the range of the same magnitude order as the experimental data and 15 outside, which represents an improvement of 6 isotopes compared with GTDB1. This shows that there is a significant gain of $6.4 \%$ in the results when we include experimental data in the parameter $\sigma_{N}$, which indicates that is better to employ available experimental values instead of adjusted theoretical estimations.

For completeness, we present in Fig. 3 our results for the $\beta^{-}$half-lives obtained with the GTBD1 and GTBD2 methods, for the cobalt and manganese families as an example. Our results are compared with experimental data and also with results obtained within microscopic models like shell model (SM) and quasiparticle random phase approximation (QRPA) from Ref. [10], and the extended 

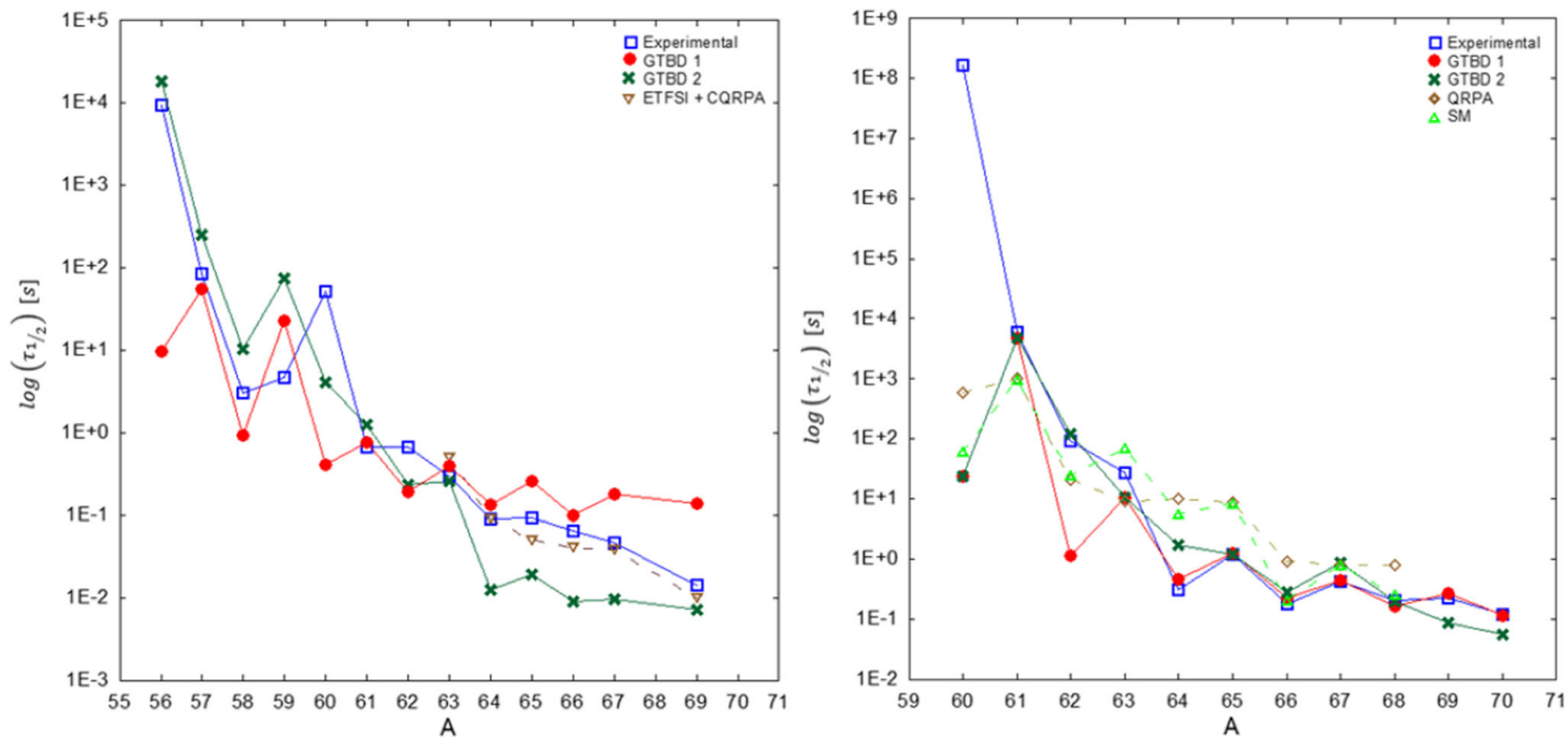

Fig. 3 Comparison of $\beta^{-}$half-lives for manganese (left panel) and cobalt (right panel) families: GTBD1 (red circles), GTBD2 (green crosses), QRPA and SM from Ref. [10], and ETFSI+CQRPA from Ref. [11]. Experimental data (blue squares) from Letter of Nuclide [5]

Thomas-Fermi plus Strutinsky integral method combined with continuum QRPA (ETFSI + CQRPA) from Ref. [11]. We observe that for both, cobalt and manganese families, the GTBD2 gives sometimes better than the GTBD1, although for other nuclei within that same family it gives worse or sometimes very similar results to those of GTBD1. From the right panel in Fig. 3 we observe that the GTBD gives better results than QRPA for cobalt. Besides, they are similar and still better than those obtained within the SM. For manganese, we observe that the half-lives evaluated within the ETFSI + CQRPA for the isotopes $63 \leq A \leq 69$ are better than our results obtained with the GTBD model.

\section{Conclusions}

We improved the results obtained for the $\beta^{-}$-decay rates within the Gross Theory of Beta Decay by using new values of the parameter $\sigma_{N}$ related to the standard deviation for the Gamow-Teller resonance. We have shown that the substitution of the parameters adjusted in Ref. [1] by minimization of the $\chi^{2}$-function by experimental data in GTBD improves the results for $\beta^{-}$-decay rates by $6.4 \%$. Also, they are sometimes better and sometimes very similar to those obtained with some systematic microscopic calculations. Despite this, we have shown that the results obtained for some particular isotopes may not be as satisfactory. Search for new improvements it will remain a motivation for our future resssearch.
Acknowledgments C.B. is a fellow of the CONICET, CCT La Plata (Argentina). R.A.M. thanks the Universidade Estadual do Sudoeste da Bahia-UESB for granting the scholarship and infrastructure.

\section{References}

1. D.N. Possidonio, R.C. Ferreira, A.J. Dimarco, C.A. Barbero, A.R. Samana, M.R. Azevedo, C.L. Santana, A.E. Mariano, Braz. J. Phys. 48, 485 (2018)

2. M.R. Azevedo, R.C. Ferreira, A.J. Dimarco, C.A. Barbero, A.R. Samana, D.N. Possidonio, Braz. Jour. Phys. https://doi.org/10.1007/s13538-019-00723-z (2019)

3. R.C. Ferreira, A.J. Dimarco, A.R. Samana, C. Barbero, Astr. J. 784:24, 1 (2014)

4. K. Takanahashi, M. Yamada, Prog. Theor. Phys. 41, 1470 (1969)

5. Letter of Nuclide, available at: https://www-nds.iaea.org/relnsd/ vcharthtml/VChartHTML.html (2019)

6. M.B. Aufderheide, I. Fushiki, S.E. Woosley, D.H. Hartmann, Astr. J. Suppl. 91, 389 (1994)

7. K. Nakayama, A.P. Galeão, F. Krmpotić, Phys. Lett. B 114, 217 (1982)

8. K. Nakayama, A.P. Galeão, F. Krmpotić, Nucl. Phys. A 399, 478 (1983)

9. A.R. Samana, C. Barbero, S.B. Duarte, A.J. Dimarco, F. Krmpotic, J. Phys. 10, 1 (2008)

10. K. Kar, A. Ray, S. Sarkar, Astron. J. 434, 662 (1994)

11. I.N. Borzov, S. Goriely, Phys. Rev. C 62, 035501 (2000)

Publisher's Note Springer Nature remains neutral with regard to jurisdictional claims in published maps and institutional affiliations. 